\title{
Autologous Immune Enhancement Therapy in Recurrent Ovarian Cancer with Metastases: A Case Report
}

\author{
Sadananda Rao Manjunath $^{\mathrm{a}}$ Ganapathi Ramanan $^{\mathrm{b}}$ \\ ${\text { Vidyasagar Devaprasad Dedeepiya }{ }^{a} \text { Hiroshi Terunuma }}^{d}$ \\ Xuewen Deng $^{d}$ Subramani Baskar ${ }^{a}$ Rajappa Senthilkumar ${ }^{a}$ \\ Paramasivam Thamaraikannan $^{a}$ Thangavelu Srinivasan $^{a}$ \\ Senthilkumar Preethy ${ }^{a, c}$ Samuel J.K. Abraham ${ }^{a, e}$ \\ ${ }^{a} \mathrm{Nichi}$-In Centre for Regenerative Medicine (NCRM), ${ }^{\mathrm{b}}$ Cancer Research and Relief \\ Trust, and ${ }^{\mathrm{C}}$ Hope Foundation (Trust), Chennai, India; ${ }^{\mathrm{d}}$ Biotherapy Institute of \\ Japan, Tokyo, and ${ }^{\mathrm{e}}$ Yamanashi University School of Medicine, Chuo, Japan
}

\section{Key Words}

Autologous immune enhancement therapy $\cdot$ Recurrent ovarian carcinoma $\cdot$ Natural killer cells (CD3-CD56+) · Activated T lymphocytes (CD3+CD56+)

\begin{abstract}
Current therapeutic modalities for ovarian cancer such as chemotherapy, radiotherapy and surgery have been reported to yield only marginal success in improving survival rates of patients and have associated adverse effects. We report here a case of recurrent stage IV ovarian cancer, treated with cell-based autologous immune enhancement therapy (AIET) along with chemotherapy and followed up for 18 months. A 54-year-old female was diagnosed with a recurrence of ovarian carcinoma 1 year after initial surgical removal followed by chemotherapy for stage IIIC ovarian carcinoma. When diagnosed in 2010 with recurrence, she had liver and spleen metastases with a CA-125 level of $243 \mathrm{U} / \mathrm{ml}$ and a stage IV clinical status. Six infusions of AIET using autologous in vitro expanded and activated natural killer (NK) cells (CD3-CD56+) and activated T lymphocytes (CD3+CD56+) were administered in combination with 6 cycles of chemotherapy with carboplatin and doxorubicin. Following this treatment, CA-125 decreased to $4.7 \mathrm{U} / \mathrm{ml}$ along with regression of the metastatic lesions and an improved quality of life. No adverse reactions were reported after the AIET transfusions. Eighteen months of follow-up revealed a static nonprogressive disease. Combining AIET with chemotherapy and other conventional treatments has been found to be effective in our experience, as reported earlier, even in patients with advanced ovarian cancer, and we recommend this strategy be considered in treating similar cases.
\end{abstract}




\section{Introduction}

Ovarian cancer ranks fifth among the leading causes of cancer-related deaths in females [1], and $75 \%$ of women are in an advanced stage of the disease when diagnosed. The prognosis after conventional treatment modalities has been poor, with a 5-year survival rate of less than 30\% [2]. Even after surgical removal of the tumor, there is still a risk of residual disease; thus, radiation and chemotherapy are administered, though both have various side effects. Treatment of recurrent ovarian cancer is only palliative, employing chemotherapeutic agents, but the adverse reactions associated with chemotherapy are many [1].

Most recently, immunotherapy has been of major interest in order to potentiate a multipronged approach to cancer. Better prognosis has been reported when chemotherapy is combined with immunotherapy than with chemotherapy alone [3]. Cell-based immunotherapy has been in practice for the past 2 decades, with published randomized clinical trials establishing its safety and efficacy in different cancers [4, 5]. Fujita et al. [6] have reported that in patients with epithelial ovarian cancers treated with T cell-based immunotherapy and chemotherapy, the 3-year disease-free interval was $82.1 \%$, while it was $54.5 \%$ in patients treated with chemotherapy alone.

Herein we report an 18-month follow-up of a patient who had recurrence of ovarian carcinoma and metastases and was treated with autologous immune enhancement therapy (AIET) in addition to palliative chemotherapy.

\section{Case Presentation}

A 54-year-old female presented with gross ascites in April 2009. A CT scan and biopsy revealed an ovarian malignancy with multiple peritoneal deposits, lesions in the left lobe of the liver and a CA-125 of $237 \mathrm{U} / \mathrm{ml}$. She was administered 3 cycles of intravenous chemotherapy with paclitaxel $(290 \mathrm{mg})$ and carboplatin (450 mg). She underwent an ovarian laparotomy; panhysterectomy, pelvic peritonectomy, omentectomy, bilateral pelvic lymph node dissection, para-aortic dissection, right sub diaphragmatic peritonectomy, appendicectomy along with excision of peritoneal nodes, and insertion of an intraperitoneal chemo port were performed in June 2009. Biopsy, post-abdominal hysterectomy with bilateral sapling oophorectomy revealed residual papillary serous cystadenocarcinoma. After the surgery, 3 cycles of intraperitoneal chemotherapy with paclitaxel $(290 \mathrm{mg})$ and cisplatin $(120 \mathrm{mg})$ were administered, once every 21 days. A CT scan in November 2009 revealed no visible signs of cancer, and the CA-125 value was $7 \mathrm{U} / \mathrm{ml}$. In July 2010, a recurrence was diagnosed following an increased CA-125 level of $243 \mathrm{U} / \mathrm{ml}$. A CT scan revealed hypoattenuating lesions in the liver, the largest one measuring $2.8 \times 2.2 \mathrm{~cm}$, lesions in the spleen, ill-defined hypodense lesions along the greater curvature of the stomach body and in the perisplenic region, small hypodense lesions noted between the medial aspect of the liver and stomach, the largest one measuring $11 \times 7 \mathrm{~mm}$, and an ovoid soft-tissue density lesion measuring $2.6 \times 1.8 \mathrm{~cm}$ in the right inguinal region. Six cycles of chemotherapy with doxorubicin (50 $\mathrm{mg}$ ) and carboplatin (450 $\mathrm{mg}$ ) along with intermittent infusions of cell-based AIET were the recommended treatment.

After informed consent was obtained, peripheral blood was collected in heparin tubes and transported to the lab under cold conditions. The in vitro cell processing and expansion were performed in a GMP-compliant lab. The peripheral blood was first centrifuged to separate the autologous plasma in the supernatant. The pellet containing white and red blood cells was mixed using phosphate buffered saline, and the routine procedure of density gradient centrifugation was done using LymphoPrep solution (GIBCO-BRL, Gaithersburg, Md., USA) to isolate the peripheral blood mononuclear cells (PBMNCs). The cell count was estimated using the Trypan blue dye-exclusion method and the cells were subjected to expansion as per the protocols described earlier [7, 8] for a period of 10-16 days. Before cell transplantation, cells were tested for endotoxin levels using a Limulus Amebocyte Lysate kit (Charles 
River Laboratories, India), and flow cytometry analysis was done to determine the percentage of natural killer (NK) cells and activated T Lymphocytes employing markers viz., CD3, CD16 and CD56.

The cell count of the isolated PBMNCs ranged between $34.8 \times 10^{6}$ and $111 \times 10^{6}$ cells, which showed a marginal decrease on day 3 , and after day 5 the cells started to increase in a steady state, reaching up to a maximum of $1.68 \times 10^{8}$ cells. Average cell count after the in vitro expansion was $1.2 \times$ $10^{8}$ cells. The percentage of NK cells (CD3-CD56+) and activated T cells (CD3+ CD56+) increased substantially after the in vitro expansion with NK cells (CD3-CD56+), increasing from 2.43 to $61.3 \%$ within total lymphocytes, and activated T cells (CD3+CD56+) increasing from 3.73 to $60.8 \%$ within total $\mathrm{T}$ lymphocytes as evaluated by flow cytometry.

Following 6 AIET transfusions given intravenously along with chemotherapy, CA-125 showed a considerable decrease to $4.7 \mathrm{U} / \mathrm{ml}$ as depicted in fig. 1. A CT scan taken in December 2010 showed a regression of the lesions in the spleen and perisplenic peritoneal deposits, stable hepatic lesions and resolution of perigastric peritoneal deposits (fig. 2). There was a marked decrease in the size of the inguinal lymph nodes from $2.6 \times 1.8$ to $1.2 \times 1.1 \mathrm{~cm}$ with no recurrence in the pelvic region. The patient showed improvements in appetite and quality of life. There were no adverse reactions following the AIET infusions. The latest follow-up in December 2011 revealed static nonprogressive disease with all the parameters, including tumor markers within normal range.

\section{Discussion}

AIET involves the in vitro expansion of NK cell (CD3-CD56+) and activated T cells $(\mathrm{CD} 3+\mathrm{CD} 56+)$ and their intravenous transfusion. Autologous immune cells intravenously administered to ovarian cancer patients have resulted in improved survival rates compared to controls, as reported by Fujita et al. [6]. Progression-free survival after chemotherapy in recurrent ovarian cancer patients ranges from 17 to 24 months, but the associated treatment-related toxicities are high. Adverse reactions commonly associated with chemotherapeutic agents such as carboplatin, gemcitabine, paclitaxel, epirubicin, doxorubicin, topotecan, etc., given in patients with recurrent ovarian cancer are anemia, leucopenia, neutropenia, thrombocytopenia, nausea, vomiting, diarrhea, constipation, neurotoxicity and alopecia [9]. This patient, in whom AIET was given in combination with chemotherapy, subjectively reported fewer or no adverse effects mentioned above. The blood counts continued to be normal and the patient had an improved appetite and quality of life after the AIET. A significant decrease in the level of CA-125 from 243 to $4.7 \mathrm{U} / \mathrm{ml}$ along with regression of the lesions and a prolonged static nonprogressive disease for more than 18 months in a case of recurrent stage IV ovarian cancer was experienced by our patient without any adverse reactions following AIET combined with chemotherapy. Also, it has been previously reported that NK cell activity enhances the action of carboplatin in the treatment of ovarian cancer [10], thus making AIET in combination with chemotherapy an attractive strategy for ovarian cancers.

Therefore, in our experience, AIET, which was combined with chemotherapy in this patient with stage IV recurrent ovarian cancer, did not have any adverse reactions demonstrating its safety. The gradual decrease in CA- 125 , regression of the metastatic lesions, improved quality of life and a prolonged duration of survival for more than 18 months with static nonprogressive lesions confirm the efficacy, which is in line with reports published earlier on cell-based immunotherapies. With this, we recommend that AIET may be considered in combination with chemotherapy and other modalities of treatment in similar cases. It is also preferable to start AIET earlier, before chemotherapy, when the peripheral blood cell counts are normal. 


\section{Acknowledgements}

The authors acknowledge M/S Hope Foundation (Trust), India, for funding the study and M/S Chennai Cell Cluster for technical advice.

\section{Disclosure Statement}

The authors have no conflicts of interest to declare.

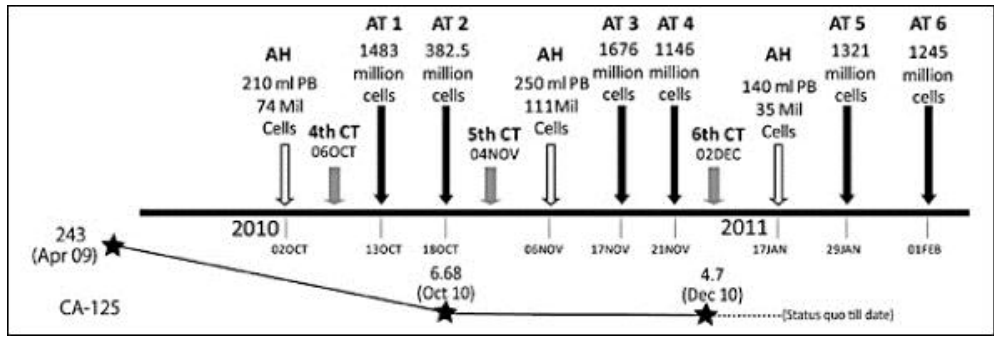

Fig. 1. Course of the treatment of 6 cycles of AIET given intermittently with chemotherapy. The decrease in CA-125 values during the course of the treatment is also depicted. $\mathrm{AH}=$ AIET peripheral blood harvest; AT = AIET transfusions; CT = chemotherapy.
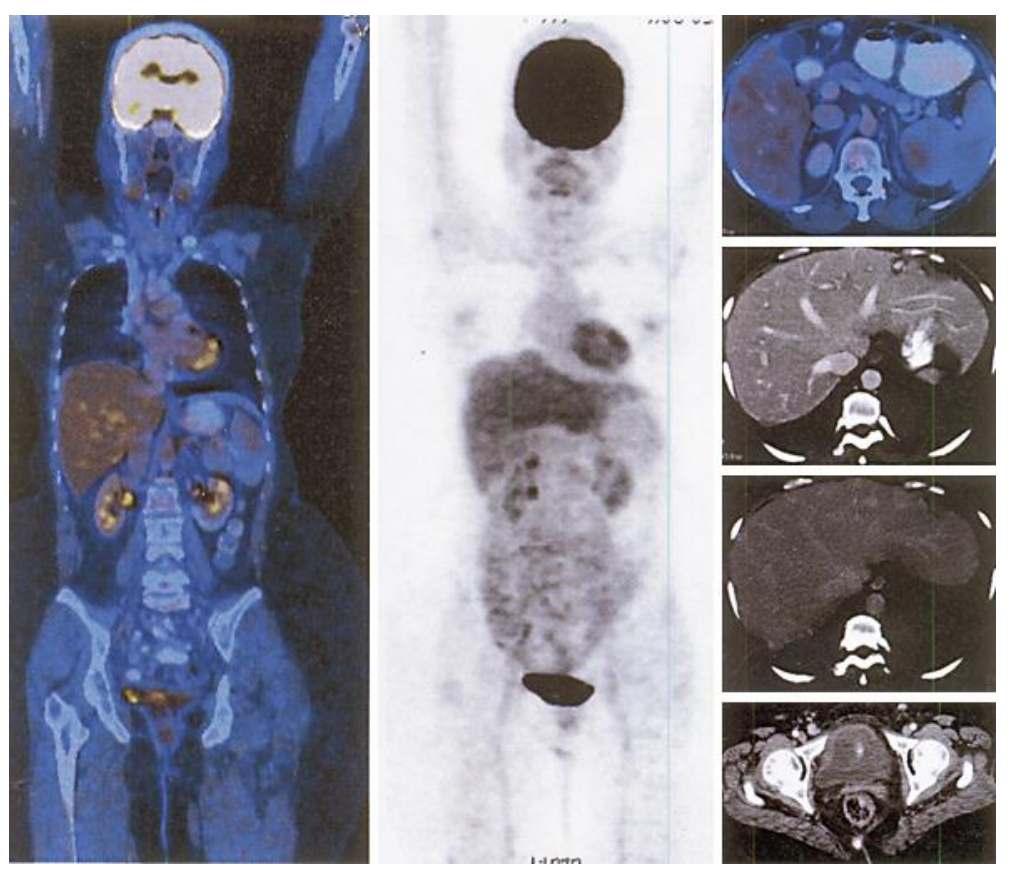

Fig. 2. CT scan of the patient taken after 3 cycles of immunotherapy and 6 cycles of chemotherapy in December 2010, showing static non-progressive lesions. 


\section{References}

1 Herzog TJ: Recurrent ovarian cancer: how important is it to treat to disease progression? Clin Cancer Res 2004;10:7439-7449.

-2 Modugno F, Ovarian Cancer and High-Risk Women Symposium Presenters: Ovarian cancer and high-risk women-implications for prevention, screening, and early detection. Gynecol Oncol 2003;91:15-31.

3 Lake RA, Robinson BW: Immunotherapy and chemotherapy - a practical partnership. Nat Rev Cancer 2005;5:397-405.

-4 Takayama T, Sekine T, Makuuchi M, Yamasaki S, Kosuge T, Yamamoto J, Shimada K, Sakamoto M, Hirohashi S, Ohashi Y, Kakizoe T: Adoptive immunotherapy to lower postsurgical recurrence rates of hepatocellular carcinoma: a randomised trial. Lancet 2000;356:802-807.

5 Kono K, Takahashi A, Ichihara F, Amemiya H, Iizuka H, Fujii H, Sekikawa T, Matsumoto Y: Prognostic significance of adoptive immunotherapy with tumor-associated lymphocytes in patients with advanced gastric cancer: a randomized trial. Clin Cancer Res 2002;8:1767-1771.

6 Fujita K, Ikarashi H, Takakuwa K, Kodama S, Tokunaga A, Takahashi T, Tanaka K: Prolonged disease-free period in patients with advanced epithelial ovarian cancer after adoptive transfer of tumor-infiltrating lymphocytes. Clin Cancer Res 1995;1:501-507.

-7 Takada M, Terunuma H, Deng X, Dewan MZ, Saji S, Kuroi K, Yamamoto N, Toi M: Refractory lung metastasis from breast cancer treated with multidisciplinary therapy including an immunological approach. Breast Cancer 2011;18:64-67.

8 Dedeepiya V, Terunuma H, Deng X, Baskar S, Manjunath S, Senthilkumar R, Murugan P, Thamaraikannan $P$, Srinivasan T, Preethy S, Abraham SJK: A comparative analysis of in vitro expansion of natural killer cells of a patient with autoimmune haemolytic anaemia and ovarian cancer with patients with other solid tumours. Oncology Letters 2011;3:435-440.

-9 Fung-Kee-Fung M, Oliver T, Elit L, Oza A, Hirte HW, Bryson P: Optimal chemotherapy treatment for women with recurrent ovarian cancer. Curr Oncol 2007;14:195-208.

10 El-Gazzar A, Perco P, Eckelhart E, Anees M, Sexl V, Mayer B, Liu Y, Mikulits W, Horvat R, Pangerl T, Zheng D, Krainer M: Natural immunity enhances the activity of a DR5 agonistic antibody and carboplatin in the treatment of ovarian cancer. Mol Cancer Ther 2010;9:1007-1018. 